TRANSACTIONS OF THE

AMERICAN MATHEMATICAL SOCIETY

Volume 352, Number 2, Pages 679-688

S 0002-9947(99)02046-2

Article electronically published on September 17, 1999

\title{
TWO SPECIAL CASES OF GANEA'S CONJECTURE
}

\author{
JEFFREY A. STROM
}

\begin{abstract}
Ganea conjectured that for any finite CW complex $X$ and any $k>0, \operatorname{cat}\left(X \times S^{k}\right)=\operatorname{cat}(X)+1$. In this paper we prove two special cases of this conjecture. The main result is the following. Let $X$ be a $(p-1)$ connected $n$-dimensional CW complex (not necessarily finite). We show that if $\operatorname{cat}(X)=\left\lfloor\frac{n}{p}\right\rfloor+1$ and $n \not \equiv-1 \bmod p($ which implies $p>1)$, then $\operatorname{cat}\left(X \times S^{k}\right)=$ $\operatorname{cat}(X)+1$. This is proved by showing that $\operatorname{wcat}\left(X \times S^{k}\right)=\operatorname{wcat}(X)+1$ in a much larger range, and then showing that under the conditions imposed, $\operatorname{cat}(X)=\operatorname{wcat}(X)$. The second special case is an extension of Singhof's earlier result for manifolds.
\end{abstract}

\section{INTRODUCTION}

For any CW complex $X$, and any $k>0$

$$
\operatorname{cat}(X) \leq \operatorname{cat}\left(X \times S^{k}\right) \leq \operatorname{cat}(X)+1
$$

(see [6], Proposition 2.3). Ganea conjectured that $\operatorname{cat}\left(X \times S^{k}\right)=\operatorname{cat}(X)+1$ for any finite complex $X$ and any $k>0$ (see [2], Problem 2).

Singhof proved the conjecture for $X$ a compact differentiable or PL manifold and $k$ lying in a bounded (possibly empty) interval depending on the dimension, connectivity and category of $X$ (see [9], Corollary 6.7 or [6], Theorems 1 and 2). More recently, Hess proved that the conjecture holds for simply connected spaces when cat is replaced with cat $_{0}$, the rational version of cat, and $k>1$ (see [4], Theorem 3). It follows that the conjecture holds for simply connected rational spaces and $k>1$.

In this paper, we will prove the following.

Theorem 3.2. Let $X$ be a $(p-1)$-connected $n$-dimensional $C W$ complex. If $\operatorname{cat}(X)$ $=\left\lfloor\frac{n}{p}\right\rfloor+1$ (i.e. $\operatorname{cat}(X)$ is as large as possible) and $n \not \equiv-1 \bmod p$ (which forces $p>1)$, then

$$
\operatorname{cat}\left(X \times S^{k}\right)=\operatorname{cat}(X)+1
$$

for all $k>0$.

This follows easily from two subsidiary results, which are interesting on their own.

Theorem 2.2. If $\operatorname{cat}(X)=\left\lfloor\frac{n}{p}\right\rfloor+1$ and $n \not \equiv-1 \bmod p$, then

$$
\operatorname{cat}(X)=\operatorname{wcat}(X) \text {. }
$$

Received by the editors January 23, 1997.

1991 Mathematics Subject Classification. Primary 55M30, 55P50; Secondary 55P42.

(C)1999 American Mathematical Society 
The proof is a simple application of the Blakers-Massey Excision Theorem. In [6], James stated that $\operatorname{cat}(X)$ and wcat $(X)$ agree in a stable range, but did not give any details. We assume that this is the result he had in mind.

Theorem 3.1. If $\mathrm{wcat}(X) \geq \frac{n+2}{2 p}+1$, then

$$
\operatorname{wcat}\left(X \times S^{k}\right) \geq \operatorname{wcat}(X)+1
$$

for all $k>0$.

With one exception, our result is the first general result on this problem which allows $X$ to be a CW complex without any additional structure (as opposed to a manifold or a rational space). Also, our result holds for arbitrarily large values of $k$, in contrast to Singhof's theorem.

The exception is the following. In [6] it is proved that if $X$ is $(p-1)$-connected with $\operatorname{cat}(X)=N$ and $\operatorname{dim}(X)=(N-1) p$, then the $(N-1)^{\text {st }}$ power of the fundamental class $\iota \in H^{p}\left(X ; \pi_{p}(X)\right)$ is nonzero. It follows that the conjecture holds in this case.

The proof of Theorem 3.1 can be interpreted in terms of product length in an appropriate cohomology theory. Let $E$ and $S_{k}$ denote the suspension spectra of $X$ and $S^{k}$, respectively, and let $\alpha \in \widetilde{E}^{*}\left(X \times S^{k}\right)$ and $\beta \in \widetilde{S}_{k}^{*}\left(X \times S^{k}\right)$ be the stable classes of $p_{1}: X \times S^{k} \longrightarrow X$ and $p_{2}: X \times S^{k} \longrightarrow S^{k}$ respectively. Then

$$
\overbrace{\alpha \wedge \cdots \wedge \alpha}^{N-1} \wedge \beta \neq 0 \in\left(E^{(N-1)} \wedge S_{k}\right)^{*}\left(X \times S^{k}\right) .
$$

The generalized cohomology version of the classical lower bound on (weak) category given by cup length now finishes the proof.

It is interesting to observe that our proof comes from an analysis of the relationship between category and weak category, while Singhof's proof arises from an examination of the relationship between category and geometric (or strong) category.

In section 4, we will show how the following result for manifolds follows directly from Singhof's theorem.

Corollary 4.2. Let $X$ be a compact differentiable or PL manifold which is $(p-1)$ connected and $n$-dimensional, with $n \geq 4$ and $\operatorname{cat}(X) \geq 4$. If

$$
\operatorname{cat}(X) \geq \frac{n+4 p+3}{2 p}
$$

then

$$
\operatorname{cat}\left(M \times S^{k}\right)=\operatorname{cat}(M)+1
$$

for every $k>1$. If $p=1$, then we may take $k=1$ as well.

Thus, applying Theorem 3.2 to a manifold improves on Singhof's result if $X$ is simply connected and $k=1$, or if $n \leq 4 p-1$. For example, if $X=S p(2)$, Singhof's theorem does not apply, but Theorem 3.2 does apply. It follows that $\operatorname{cat}\left(S p(2) \times S^{k}\right)=5$ for all $k>0$.

I have recently received a preprint of a paper by Yu Rudyak [8], in which he gives a result similar to Corollary 4.2 , with a similar proof.

It is important to point out that since this paper was written, Norio Iwase [5] has found counterexamples to Ganea's conjecture. Intuitively, the proof of our main theorem rests on the idea that the obstruction to lifting the diagonal map of $X \times S^{k}$ 
into $T^{N}\left(X \times S^{k}\right)$ is a $k$-fold suspension of the obstruction to lifting the diagonal map of $X$ into $T^{N-1} X$. Iwase also noticed this and, roughly speaking, constructed spaces $X$ for which the latter obstruction is stably trivial.

I'd like to thank Edward Fadell, Sufian Husseini, and Doug Lepro for their help in preparing this paper. I am also grateful to Alejandro Adem for pointing out that the proof Theorem 3.2 does not require the use of generalized cohomology theories.

\section{Preliminaries}

In this section, we will describe our notation and recall some basic facts concerning the Lusternik-Schnirelman category.

1.1. Notation. Throughout this paper, the space $X$ is a (not necessarily finite) pointed CW complex. We denote the set of pointed homotopy classes of maps $f: X \longrightarrow Y$ by $[X, Y]$. We denote the smash product of 2 spaces (or spectra) by $A \wedge B$. The $N$-fold smash product of $A$ with itself is

$$
A^{(N)}=\overbrace{A \wedge A \wedge \cdots \wedge A}^{N} .
$$

We will never refer explicitly to the skeleta of $X$, so this will not cause any confusion. The canonical projection

$$
X_{1} \times X_{2} \times \cdots \times X_{N} \longrightarrow X_{1} \wedge X_{2} \wedge \cdots \wedge X_{N}
$$

is denoted by $\wedge$. The composition $\wedge \circ f$ will be abbreviated $\hat{f}$.

1.2. Lusternik-Schnirelman category. Next, we recall some basic facts about Lusternik-Schnirelman category. For a more complete survey, see [5].

Definition. Let $X$ be a CW complex with base point $*$. Write

$$
T^{N} X=\left\{\left(x_{1}, \ldots, x_{n}\right) \mid x_{i}=* \text { for some } i\right\} \subseteq X^{N} .
$$

The category of $X$ (denoted $\operatorname{cat}(X))$ is the least integer $N$ such that there is a lift up to homotopy in the diagram

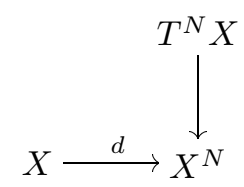

There is a related notion, the weak category of $X$ (see [3]); wcat $(X)$ is the least $N$ so that $\hat{d}: X \longrightarrow X^{(N)}$ is nullhomotopic. Since $X^{(N)}=X^{N} / T^{N} X$, $\operatorname{cat}(X) \geq$ $\operatorname{wcat}(X)$.

It follows immediately from the cellular approximation theorem that if $X$ is $n$ dimensional and $(p-1)$-connected, then $\operatorname{cat}(X) \leq\left\lfloor\frac{n}{p}\right\rfloor+1$ (see [6], Proposition $5.1)$.

Note 1.1. The conditions that appear in our theorems are of the form $\operatorname{cat}(X) \geq$ $\frac{n+2}{p}$. An easy numerical argument shows that for an integer $N$

$$
\frac{n+2}{p} \leq N \leq\left\lfloor\frac{n}{p}\right\rfloor+1
$$

if and only if $N=\left\lfloor\frac{n}{p}\right\rfloor+1$ and $n \not \equiv-1 \bmod p$. 
1.3. Homotopy classes of maps. Recall that a map $\phi: S \longrightarrow T$ of pointed sets is weakly injective if $\phi(s)=*$ if and only if $s=*$.

Lemma 1.2. Let $\wedge: X \times Y \longrightarrow X \wedge Y$. Then

$$
\wedge^{*}:[X \wedge Y, Z] \longrightarrow[X \times Y, Z]
$$

is weakly injective for any space $Z$.

Proof. The sequence

$$
[X \times Y, Z] \stackrel{\wedge^{*}}{\longleftarrow}[X \wedge Y, Z] \stackrel{\partial^{*}}{\longleftarrow}[\Sigma(X \vee Y), Z] \stackrel{(\Sigma i)^{*}}{\longleftarrow}[\Sigma(X \times Y), Z]
$$

is an exact sequence of pointed sets (see [12], Theorem 2.41). Therefore, it suffices to show that $\partial^{*}=0$, or equivalently $(\Sigma i)^{*}$ is surjective.

Since the suspension functor $\Sigma$ and the loop space functor $\Omega$ are adjoint (see [12], Corollary 2.8), we have the commutative diagram

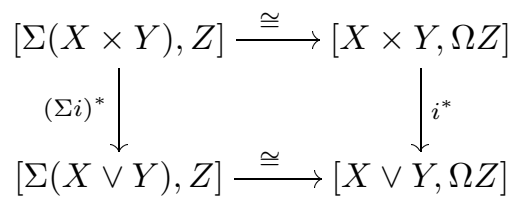

Thus it suffices to show that $i^{*}$ is surjective.

Recall that $\Omega X$ is an $H$ space with multiplication given by juxtaposition of loops (see [12], Example 2.15). Given $f \vee g: X \vee Y \longrightarrow \Omega X$, define $h: X \times Y \longrightarrow \Omega Z$ by the formula $h(x, y)=f(x) \cdot g(y)$. Then

$$
i^{*}(h)=h \circ i=\left.h\right|_{X \vee Y} \simeq f \vee g .
$$

This proves the lemma.

\section{Category and Weak Category}

In this section, we will give a condition under which $\operatorname{cat}(X)=\operatorname{wcat}(X)$. The key ingredient is Lemma 2.1, which is equivalent to the Blakers-Massey Excision Theorem.

Lemma 2.1. Let $A \longrightarrow X \stackrel{j}{\longrightarrow} B$ be a cofibration, and let $F \longrightarrow Y \stackrel{q}{\longrightarrow} B$ result from converting $j$ to a fibration. Assume $A, X$, and $B$ are simply connected. Then there is a commutative diagram

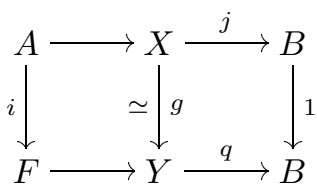

If $A$ is $(a-1)$-connected, and $B$ is $(b-1)$-connected, then $i$ is an $(a+b-2)$ equivalence.

Proof. The commutativity of the diagram yields an infinite commutative ladder

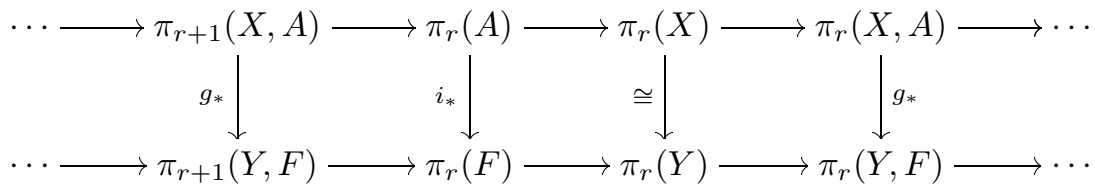


By the 5 lemma it suffices to show that $g_{*}$ is an $(a+b-1)$-equivalence.

We have the diagram

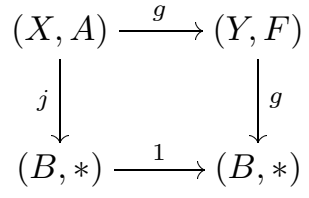

in which $q$ is a homotopy isomorphism. Since all spaces involved are simply connected, $j$ is an $(a+b-1)$-equivalence by the Blakers-Massey Excision Theorem (Theorem I of [1]). It follows that $g$ is an $(a+b-1)$-equivalence.

Now we can prove the main theorem of this section.

Theorem 2.2. Let $X$ be an $n$-dimensional $(p-1)$-connected $C W$ complex. If $\operatorname{cat}(X)=\left\lfloor\frac{n}{p}\right\rfloor+1$ and $n \not \equiv-1 \bmod p$ (which forces $\left.p>1\right)$, then

$$
\operatorname{cat}(X)=\operatorname{wcat}(X) \text {. }
$$

Proof. Write $N=\operatorname{cat}(X)$. By Note 1.1 , the condition on $\operatorname{cat}(X)$ is equivalent to $N \geq \frac{n+2}{p}$.

Let $F \longrightarrow E \longrightarrow X^{(N-1)}$ result from converting the map $\wedge: X^{N-1} \longrightarrow X^{(N-1)}$ to a fibration. Then we have the diagram

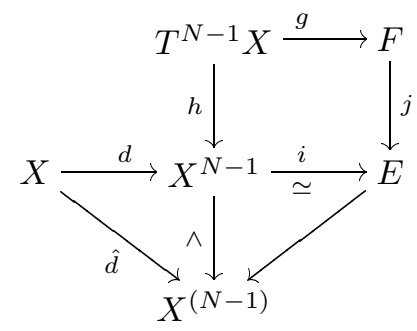

Since $T^{N-1} X$ is $(p-1)$-connected and $X^{(N-1)}$ is $((N-1) p-1)$-connected, $g$ is an

$$
(N-1) p+p-2=N p-2
$$

equivalence by Lemma 2.1. It follows that $g_{*}:\left[X, T^{N-1} X\right] \longrightarrow[X, F]$ is a surjection because

$$
n=\left(\frac{n+2}{p}\right) p-2 \leq N p-2 .
$$

Suppose wcat $(X)<\operatorname{cat}(X)$. Then $\hat{d} \simeq *$, and so there is a lift (up to homotopy) $\lambda$ of $i \circ d$ into $F$. Let $\kappa \in\left(g_{*}\right)^{-1}(\lambda)$. Then

$$
i \circ h \circ \kappa \simeq j \circ g \circ \kappa=j \circ \lambda \simeq i \circ d .
$$

Since $i$ is a homotopy equivalence, it follows that $h \circ \kappa \simeq d$; that is, $\operatorname{cat}(X)<N$. This is a contradiction.

\section{The Main Theorem}

In this section and the next, we assume that $X$ is a $(p-1)$-connected $n$ dimensional CW complex. 
Theorem 3.1. If $\operatorname{wcat}(X) \geq \frac{n+2}{2 p}+1$, then

$$
\operatorname{wcat}\left(X \times S^{k}\right) \geq \operatorname{wcat}(X)+1
$$

for all $k>0$.

Proof. Write $\operatorname{wcat}(X)=N$; then the map $\hat{d}: X \longrightarrow X^{(N-1)}$ is nontrivial. Since $X^{(N-1)}$ is $((N-1) p-1)$-connected, and

$$
n=2\left(\left(\frac{n+2}{2 p}+1\right)-1\right) p-2 \leq 2(N-1) p-2
$$

this map is stably nontrivial by the Freudenthal Suspension Theorem.

We need to show the map $\hat{d}: X \times S^{k} \longrightarrow\left(X \times S^{k}\right)^{(N)}$ is nontrivial. Consider the commutative diagram

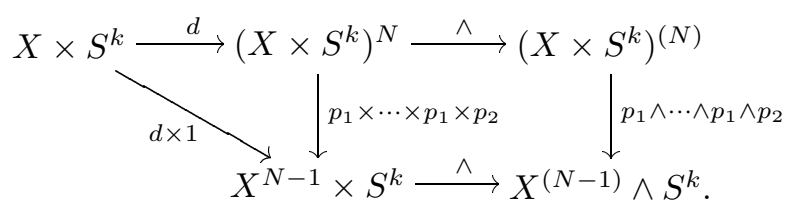

It is certainly enough to show that the composite

$$
X \times S^{k} \stackrel{d \times 1}{\longrightarrow} X^{N-1} \times S^{k} \stackrel{\wedge}{\longrightarrow} X^{(N-1)} \wedge S^{k}
$$

is nontrivial. From the commutative diagram

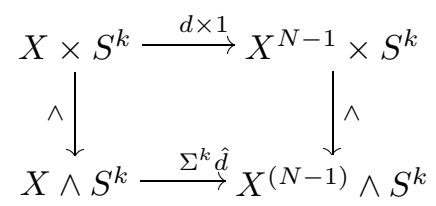

we see that it is enough to show that the composite

$$
X \times S^{k} \stackrel{\wedge}{\longrightarrow} \wedge S^{k} \stackrel{\Sigma^{k} f}{\longrightarrow} X^{(N-1)} \wedge S^{k}
$$

represents a nontrivial class in $\left[X \times S^{k}, X^{(N-1)} \wedge S^{k}\right]$. Since $\hat{d}$ is stably nontrivial,

$$
\Sigma^{k} \hat{d} \neq * \in\left[X \wedge S^{k}, X^{(N-1)} \wedge S^{k}\right] .
$$

By Lemma $1.2, \wedge^{*}$ is injective. This proves the theorem.

Remark. It is easy to view this proof in terms of product length using appropriate cohomology theories. We refer the reader to [10], Chapters 8, 9 and 13 for information about generalized cohomology.

Let $E$ and $S_{k}$ denote the suspension spectra of $X$ and $S^{k}$, respectively. Let $p_{1}: X \times S^{k} \longrightarrow X$ and $p_{2}: X \times S^{k} \longrightarrow S^{k}$ be the projections, and let $\alpha \in \widetilde{E}^{*}\left(X \times S^{k}\right)$ and $\beta \in \widetilde{S}_{k}^{*}\left(X \times S^{k}\right)$ be the stable classes of the maps $p_{1}$ and $p_{2}$, respectively.

The product

$$
\overbrace{\alpha \wedge \cdots \wedge \alpha}^{N-1} \wedge \beta \in\left(E^{(N-1)} \wedge S_{k}\right)^{*}\left(X \times S^{k}\right)
$$

is the stable homotopy class of the map

$$
\left(\left(p_{1} \wedge \cdots \wedge p_{i}\right) \wedge p_{2}\right) \circ \hat{d}: X \times S^{k} \longrightarrow X^{(N-1)} \wedge S^{k}
$$

We saw in the proof of Theorem 3.1 that this map is nontrivial.

In fact it is stably nontrivial. We have seen that $\hat{d}$ is stably nontrivial; let $\delta \in\left(E^{(N-1)} \wedge S_{k}\right)^{*}\left(X \wedge S^{k}\right)$ denote the corresponding cohomology class. Then 
$\alpha \wedge \cdots \wedge \alpha \wedge \beta=\wedge^{*} \delta$ which is nonzero by the cohomology analogue of Lemma 1.2. The existence of an $N$-fold product in the cohomology of $X \times S^{k}$ immediately implies wcat $\left(X \times S^{k}\right) \geq N+1$.

This argument is easily extended to products of spheres. Let $\sigma_{i}$ be the class of the identity map in $\left(\widetilde{S}_{k_{i}}\right)^{*}\left(S^{k_{i}}\right)$, and write

$$
\beta_{i}=p_{i+1}^{*}\left(\sigma_{i}\right) \in\left(E^{(N-1)} \wedge S_{k_{1}} \wedge \cdots \wedge S_{k_{m}}\right)^{*}\left(X \times S^{k_{1}} \times \cdots \times S^{k_{m}}\right) .
$$

It now follows just as above that $\alpha \wedge \cdots \wedge \alpha \wedge \beta_{1} \wedge \cdots \wedge \beta_{m} \neq 0$ and so

$$
\operatorname{wcat}\left(X \times S^{k_{1}} \times \cdots \times S^{k_{m}}\right) \geq \operatorname{wcat}(X)+m .
$$

Theorem 3.2. Let $X$ be a n-dimensional $(p-1)$-connected $C W$ complex. If $\operatorname{cat}(X)$ $=\left\lfloor\frac{n}{p}\right\rfloor+1$ and $n \not \equiv-1 \bmod p$ (forcing $p>1$ ), then

$$
\operatorname{cat}\left(X \times S^{k}\right)=\operatorname{cat}(X)+1
$$

for all $k>0$.

Proof. If $n<2 p$, then $\operatorname{cat}(X) \leq 2$, and the result is trivial. If $\operatorname{cat}(X)=1$, then $X \times S^{k} \simeq S^{k}$, so cat $\left(X \times S^{k}\right)=2$. If $X$ is nontrivial, there will be a nonzero class $u \in \widetilde{H}^{*}(X)$, and hence a nontrivial cup product in $H^{*}\left(X \times S^{k}\right)$. Therefore, we assume $n \geq 2 p$; it follows that $\frac{n+2}{p}>\frac{n+2}{2 p}+1$.

By Theorem 2.2, $\operatorname{cat}(X)=\operatorname{wcat}(X)$. By Note 1.1, $\operatorname{cat}(X) \geq \frac{n+2}{p}$, and so

$$
\operatorname{wcat}(X)=\operatorname{cat}(X) \geq \frac{n+2}{p} \geq \frac{n+2}{2 p}+1,
$$

so Theorem 3.1 applies. Therefore,

$$
\operatorname{cat}(X)+1 \geq \operatorname{cat}\left(X \times S^{k}\right) \geq \operatorname{wcat}\left(X \times S^{k}\right) \geq \operatorname{wcat}(X)+1=\operatorname{cat}(X)+1,
$$

and the theorem is proved.

Using the remarks following Theorem 3.1, we can extend this argument to show

$$
\operatorname{cat}\left(X \times S^{k_{1}} \times \cdots \times S^{k_{m}}\right)=\operatorname{cat}(X)+m
$$

when $X$ satisfies the hypotheses of Theorem 3.2.

Example 3.3. Let $X=S p(2)$. Then $n=\operatorname{dim}(S p(2))=10$, and $S p(2)$ is 2connected, so $p=3$. By Example 4.4 of [10], $\operatorname{cat}(S p(2))=4$. Thus

$$
\operatorname{cat}(S p(2))=4=\left\lfloor\frac{10}{3}\right\rfloor+1=4,
$$

so Theorem 3.2 applies. It follows that

$$
\operatorname{cat}\left(S p(2) \times S^{k_{1}} \times \cdots \times S^{k_{m}}\right)=4+m
$$

for all $k_{i}>0$.

\section{Extending Singhof's Theorem}

We continue to assume that $X$ is $n$-dimensional and $(p-1)$-connected.

The results of [9] and [7] which concern Ganea's conjecture are explicitly stated only for the case $p=1$. The arguments given apply to larger $p$, and significantly better results follow immediately. From this point of view, the most complete statement of Singhof's theorem is as follows (cf. [9], Theorems 6.1 and 6.2, and Corollary 6.7 or [7], Theorems 1 and 2). 
Theorem (Singhof). If $X$ is an $n$-dimensional $(p-1)$-connected compact $P L$ or differentiable manifold with $n \geq 4$, $\operatorname{cat}(X)=N \geq 4$, and

$$
\left\{\begin{array}{l}
N \geq \frac{n+4 k+3}{2 k} \quad \text { if } k \leq p, \\
N \geq \frac{n+k+3 p+3}{2 p} \quad \text { if } k \geq p,
\end{array}\right.
$$

then $\operatorname{cat}\left(X \times S^{k}\right)=N+1$.

This section is devoted to extending the values of $k$ for which Singhof's theorem applies. For the rest of this section, we assume that $X$ is a connected compact PL or differentiable manifold, that $n \geq 4$, and that $N=\operatorname{cat}(X) \geq 4$.

We will show that if Singhof's theorem applies to the manifold $X$ and $S^{p}$, then

$$
\operatorname{cat}\left(X \times S^{k}\right)=\operatorname{cat}(X)+1
$$

for all $k>1$. We first show that $\operatorname{cat}\left(X \times\left(S^{p}\right)^{M}\right)=\operatorname{cat}(X)+M$. For each $k>\frac{p}{2}$, Singhof's theorem will apply to $X \times\left(S^{p}\right)^{M}$ and $S^{k}$ if $M$ is large enough. It is then a simple matter to conclude that $\operatorname{cat}\left(X \times S^{k}\right)=\operatorname{cat}(X)+1$.

The case $k \leq \frac{p}{2}$ is more difficult. For this, we choose $M$ large enough that

$$
\operatorname{cat}\left(X \times\left(S^{p}\right)^{M} \times S^{\left\lceil\frac{p}{2}\right\rceil}\right)=\operatorname{cat}(X)+M+1
$$

and such that Singhof's theorem applies to $X \times\left(S^{p}\right)^{M} \times S^{\left\lceil\frac{p}{2}\right\rceil}$. Then the previous case shows that we may take any $k>\frac{p}{2}$. An easy induction completes the argument.

In [8], Rudyak uses Singhof's theorem as stated in [9] and an argument similar to ours to prove the case $p=1$ of our Corollary 4.2 (see [8], Theorem 3.7).

Lemma 4.1. Let $X$ be as above, and suppose

$$
N \geq \frac{n+4 p+3}{2 p} .
$$

Let $k>\frac{p}{2}$. Write $X^{\prime}=X \times\left(S^{p}\right)^{M} \times S^{k}, n^{\prime}=\operatorname{dim}\left(X^{\prime}\right), N^{\prime}=\operatorname{cat}\left(X^{\prime}\right)$, and denote the connectivity of $X^{\prime}$ by $\left(p^{\prime}-1\right)$ (of course, these may vary with $M$ ). If $M$ is large enough, then

$$
N^{\prime}=\operatorname{cat}\left(X^{\prime}\right)=N+M+1
$$

and

$$
N^{\prime} \geq \frac{n^{\prime}+4 p^{\prime}+3}{2 p^{\prime}}
$$

Proof. By Singhof's theorem applied to $X$ and $S^{p}$,

$$
\operatorname{cat}\left(X \times S^{p}\right)=\operatorname{cat}(X)+1 .
$$

Furthermore, since

$$
\operatorname{cat}\left(X \times S^{p}\right)=N+1 \geq \frac{(n+p)+4 p+3}{2 p}=\left(\frac{n+4 p+3}{2 p}\right)+\frac{1}{2},
$$

Singhof's theorem also applies to $X \times S^{p}$ and $S^{p}$. Inductively, we obtain

$$
\operatorname{cat}\left(X \times\left(S^{p}\right)^{M}\right)=N+M \text {. }
$$

We have to consider 2 cases. First assume $k \geq p$. To apply Singhof's theorem to $X \times\left(S^{p}\right)^{M}$ and $S^{k}$, we require

$$
N+M=\operatorname{cat}\left(X \times\left(S^{p}\right)^{M}\right) \geq \frac{(n+M p)+k+3 p+3}{2 p}
$$


or

$$
N \geq \frac{n+k+3 p+3}{2 p}-\frac{M}{2} .
$$

Clearly, if $M$ is large enough, this inequality will hold. The case $\frac{p}{2}<k \leq p$ is similar: we require

or

$$
N+M=\operatorname{cat}\left(X \times\left(S^{p}\right)^{M}\right) \geq \frac{(n+M p)+4 k+3}{2 k}
$$

$$
N \geq \frac{n+4 k+3}{2 k}-M\left(1-\frac{p}{2 k}\right)
$$

Since $k>\frac{p}{2}$, this inequality will hold for $M$ large enough. This proves the first assertion.

To prove the second assertion in the case $k \geq p$, we require

or

$$
N+M+1 \geq \frac{(n+M p+k)+4 p+3}{2 p}
$$

$$
N+1 \geq \frac{n+k+4 p+3}{2 p}-\frac{M}{2} .
$$

In the case $\frac{p}{2}<k \leq p$, we require

or

$$
N+M+1 \geq \frac{n+M p+k+4 k+3}{2 k}
$$

$$
N+1 \geq \frac{n+5 k+3}{2 k}-M\left(1-\frac{p}{2 k}\right) .
$$

In either case, the condition holds for $M$ large enough.

Corollary 4.2. If $X$ is as above, and $N \geq \frac{n+4 p+3}{2 p}$, then

$$
\operatorname{cat}\left(X \times S^{k}\right)=\operatorname{cat}(X)+1
$$

for all $k>1$. If $p=1$, we may allow $k=1$.

Proof. We begin by defining a decreasing sequence of positive integers by $p_{1}=p$, $p_{j+1}=\frac{p_{j}+1}{2}$ if $p_{j}$ is odd, and $p_{j+1}=\frac{p_{j}}{2}+1$ if $p_{j}$ is even. It is easy to see that if $p>1$ this sequence decreases to 2 , where it stabilizes and if $p=1$, it is constantly 1. Notice that $p_{j+1}>\frac{p_{j}}{2}$.

We now define a sequence of spaces $X_{j}$. They will be $n_{j}$-dimensional, $\left(p_{j}-1\right)$ connected, and $\operatorname{cat}\left(X_{j}\right)=N_{j}$. The key properties of the $X_{j}$ are the following:

1. $X_{j+1}=X_{j} \times\left(S^{p_{j}}\right)^{M_{j}} \times S^{p_{j+1}}$;

2. $N_{j+1}=N_{j}+M_{j}+1$;

3. $N_{j} \geq \frac{n_{j}+4 p_{j}+3}{2 p_{j}}$.

To construct the $X_{j}$, first set $X_{1}=X$. Given $X_{j}$, define $X_{j+1}$ by property 1 ; by Lemma 4.1 we can find an $M_{j}$ large enough that 2 and 3 are satisfied.

First we consider the case $p>1$. Let $j$ be large enough that $p_{j}=2$, and consider $X_{j}$. By definition,

$$
X_{j}=X \times\left(\left(S^{p}\right)^{M_{1}} \times \prod_{i=2}^{j-1}\left(S^{p_{i}}\right)^{M_{i}+1}\right) \times S^{2} .
$$

Write $S=\left(S^{p}\right)^{M_{1}} \times \prod\left(S^{p_{i}}\right)^{M_{i}+1}$ and $s=\operatorname{cat}(S)$. By property 2 , we see $N_{j}=$ $N+s+1$. 
Let $k \geq 2$. By Lemma 4.1, there is an $M$ large enough that

$$
\text { cat }\left(X_{j} \times\left(S^{2}\right)^{M} \times S^{k}\right)=N_{j}+M+1=N+s+M+2 .
$$

On the other hand,

$$
\text { cat }\left(\left(X \times S^{k}\right) \times S \times\left(S^{2}\right)^{M+1}\right) \leq \operatorname{cat}\left(X \times S^{k}\right)+s+M+1 .
$$

Together, these inequalities imply that $\operatorname{cat}\left(X \times S^{k}\right) \geq N+1$. It follows that $\operatorname{cat}\left(X \times S^{k}\right)=\operatorname{cat}(X)+1$.

The case $p=1$ is easier: we apply Lemma 4.1 directly to $X$ and argue as in the previous paragraph.

By increasing the $M_{j}$ if necessary, we can use the same argument to show that under the conditions of Corollary 4.2 ,

$$
\operatorname{cat}\left(X \times S^{k_{1}} \times \cdots \times S^{k_{m}}\right)=\operatorname{cat}(X)+m .
$$

If $p>1$, we need to require $k_{i} \geq 2$, but if $p=1$, we allow $k_{i}=1$ also. We omit the details.

Example 4.3. Let $X=S p(2)$, as in Example 3.3. Since

$$
\operatorname{cat}(S p(2))=4<\frac{10+4 \cdot 3+3}{2 \cdot 3}=\frac{25}{6},
$$

neither Singhof's theorem nor Corollary 4.2 apply to $S p(2)$.

\section{REFERENCES}

[1] Blakers and W. Massey: The homotopy groups of a triad, II. Ann. Math. (1952) 192-201. MR 13:485f

[2] T. Ganea: Some problems on numerical homotopy invariants. Lecture Notes in Mathematics 249 (1971) 23-30. MR 49:3910

[3] W. J. Gilbert: Some examples for weak category and conilpotency, Ill. J. Math. 12 (1968), 421-432. MR 37:6930

[4] K. P. Hess: A proof of Ganea's conjecture for rational spaces. Topology, 30 (1991), 205-214. MR 92d:55012

[5] N. Iwase: Ganea's conjecture on Lusternik-Schnirelmann category. Bull. London Math. Society 30 (1998), 623-634. CMP 98:17

[6] I. M. James: On category in the sense of Lusternik and Schnirelmann. Topology, 17 (1978), 331-348. MR 80i:55001

[7] L. Montejano: A quick proof of Singhof's cat $\left(M \times S^{1}\right)=\operatorname{cat}(M)+1$ theorem. Manuscripta Math., 42 (1983), 49-52. MR 85a:55002

[8] Y. Rudyak: On category weight and its applications. Topology, 38 (1999), 37-55. MR 99f: 55007

[9] W. Singhof: Minimal coverings of manifolds with balls. Manuscripta Math., 29 (1979), 385415. MR 80k:55012

[10] P. A. Schweitzer: Secondary cohomology operations induced by the diagonal mapping. Topology. 3 (1965), 337-355. MR 32:451

[11] J. Strom, Category weight and essential category weight, Ph.D thesis, University of Wisconsin (1997).

[12] R. Switzer: Algebraic Topology: Homotopy and Homology. Springer-Verlag (1975). MR 52:6695

[13] G. W. Whitehead: Elements of Homotopy Theory. Springer-Verlag (1978). MR 80b:55001

Department of Mathematics, University of Wisconsin-Madison, Madison, Wisconsin 53706

Current address: Department of Mathematics, Dartmouth College, Hanover, New Hampshire 03755

E-mail address: jeffrey.strom@dartmouth.edu 